\title{
Wegener's Granulomatosis: Extraosseous Uptake in HDP Bone Scintigraphy- A Case Report
}

\author{
Farida Yasmin, Md. Mahbub ur Rahman, Jasmine Ara Haque, Hosne Ara Rahman, Md. Abu Bakker \\ Siddique, Samira Sharmin \\ Institute of Nuclear Medicine and Allied Sciences, Mitford, Dhaka
}

Address for Correspondence: Dr. Farida Yasmin, Medical Officer, Institute of Nuclear Medicine and Allied Sciences, Sir Salimullah Medical College Campus, MITFORD, Dhaka, E-mail: farida.sb.38@gmail.com

\begin{abstract}
Bone scan is most frequently prescribed for evaluation of patients with known malignancy or screening of bony uptake for unexplained body ache. Extraosseous uptake in bone scan is point of interest because sometimes additional information could be found beside skeletal abnormality. Here we discuss an interesting case of extraosseous uptake.
\end{abstract}

Key words: Extraosseous MDP uptake, Wegener's Granulomatosis.

\section{INTRODUCTION}

Bone scintigraphy is very sensitive method for detecting various physiological and pathological status of skeletal system. Extraosseous uptake in bone scan helps in getting important findings about the patients hidden state of disease which may have remained undiagnosed otherwise (1).

\section{CASE REPORT}

A 70 years old man with complaint of generalized body ache was referred to INMAS, Mitford for whole body planar bone scan. This patient has a history of sinusitis, persistent cough and weight loss for several months. His biochemical profile showed normal serum creatinine, calcium, phosphate and parathormone level. X-ray chest showed diffuse, ill defined opacity involving all the zones of right lung and upper, mid zones of left lung, however more marked in left perihilar region (Figure.1). Tuberculin test was negative. Bone scan with Tc99m MDP showed normal radiotracer distribution in skeletal system with significant extraosseous uptake in both lungs more on left and liver (Figure 2).

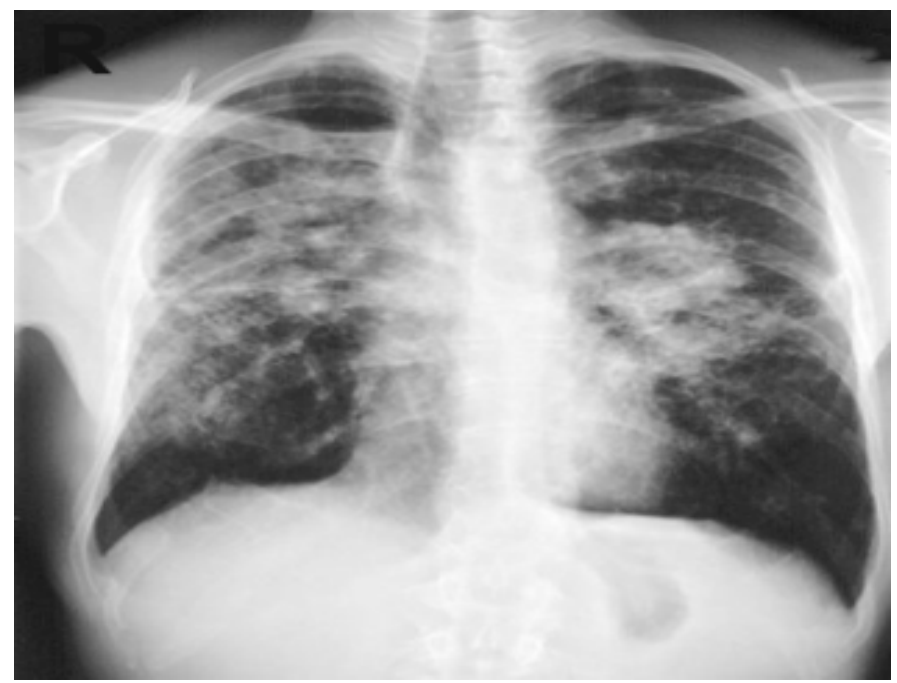

Figure1. X-ray chest showing diffuse ill-defined opacity involving both lungs

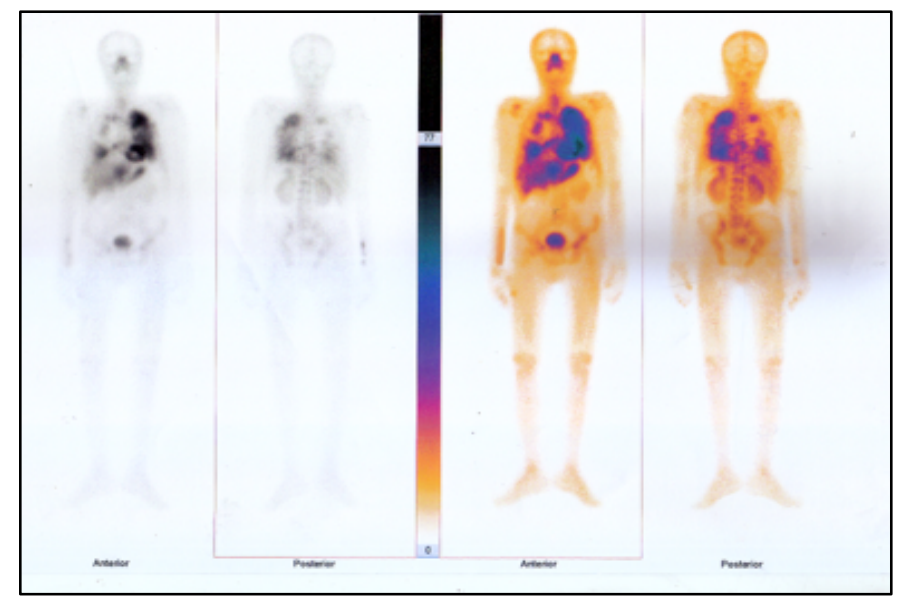

Figure 2. Tc-99m MDP bone scan showing radiotracer uptake on both lung and diffuse hepatic uptake. The skeleton shows normal tracer distribution

\section{DISCUSSION}

Radionuclide bone scan is a sensitive imaging method for diagnosis and staging of various 
malignant and benign diseases. However tracer sometime has been taken up by soft tissues and evaluating such findings may have a diagnostic value (2).

In this case the patient was sent to the institute for bone scan considering the lung findings in chest Xray. It was suspected as a case of tuberculosis, carcinoma of lung, secondary metastasis in lung or connective tissue disorder. As tuberculin test was negative, tuberculosis was excluded. In this circumstances the most common provisional diagnosis was carcinoma of lung, secondary metastasis in lungs or connective tissue disorder. As radiotracer was taken up by both lungs and liver, a CT guided FNAC of lung was recommended. CT guided FNAC showed large cavitating lung nodule revealed granulomas with geographic patterns of central necrosis and accompanying vasculitis, the granulomas were surrounded by a zone of fibroblastic proliferation with giant cells and leukocytes infiltrate. Finally it was diagnosed a case of Wegener's granulomatosis.

Wegener's granulomatosis is a disease of unknown etiology which was described for the first time by Wegener's in 1936. This disease usually present as a triad of airway necrotizing granulomatosis, systemic vasculitis and focal necrotizing granulomatosis (3). Usually clinical criteria make the diagnosis with high probability but in this case, the clinical findings did not match with typical Wegener's granulomatosis. Commonly the disease involves lung and kidney with presence of $p$ ANCA, c- ANCA and biopsy confirm the diagnosis. Bone scan has no role in the diagnosis of Wegener's granulomatosis. But there are so many reported instances where lung uptake are found in Wegener's granulamatosis $(1,3,4)$. In this case, lung uptake corresponds well to the chest radiograph abnormalities but cause of liver uptake remain uncertain. Moreover lung uptake on bone scan has been noted in various condition like metastatic calcification, fibrothroax, pleural effusion, primary lung tumor, sarcoidosis, berylliosis but not usually in Wegener's granulomatosis (5).
However, diffuse liver uptake of tracer is noted in hepatic necrosis due to Budd-Chiari syndrome, amphotericin, epirubicin, cytotoxic antibiotic treatment, cocaine treatment, ischemic hepatopathy, patients treated with high dose methotrexate (6). Those reasons for diffuse hepatic uptake did not clinically match to this patient and they were excluded. The faulty radiopharmaceutical preparation is also a cause of liver uptake (7). In this case radiopharmaceutical quality control result rechecked and found to be normal. Regarding liver uptake in this case, liver function test was performed and minor abnormality reflect hepatic damage. In this case, diffuse hepatic uptake of MDP was probably due to hepatic hypoxic necrosis resulted from respiratory failure caused by granulomatosis inflammation and necrotizing vasculitis. There are several reports where diffuse hepatic uptake occured due to respiratory failure $(7,8)$. The possible pathophysiology of increased liver uptake of Tc-99m MDP may be due to the presence of necrotic or degenerative tissue that sets free intracellular calcium and phosphorus complexes in liver (8).

\section{CONCLUSION}

The case reported here emphasizes that Wegener's granulomatosis may be include among known case of lung uptake in MDP bone scan. Apart from this, the reported cause should help the nuclear medicine physicians as well as the referring physicians to develop their awareness about the disease.

\section{REFERENCES}

1. Gnanasegaran $\mathrm{G}$, Cook $\mathrm{G}$, Adamson $\mathrm{K}$, Fogelman I. Patterns, Variants, Artifacts, and pitfalls in conventional radionuclide bone imaging and SPECT/CT. Semin Nucl Med 2009; 39: 380-395.

2. Rafique AM, Haque ul H, Fatima S, Irfan J. Liver uptake of Tc-99m MDP in metastatic breast carcinoma . Pak.J Nucl Med.2011;1:7073.

3. Wegener Granulomatosis: Case report and brief literature review. Shafiei K, Luther E, Archie 
M, Gulick J, Fowler R M . J Am Board Fam Med. 2003;vol:16; no:6;555-559.

4. Artifacts and non-ooeous uptake in bone scintigrphy . Imaging reports of 20 cases. Weiner MG, Jenicke L, Muller V, Bohuslavizki H K. Radiol Oncol . 2001;35(3) :185-91.

5. Wegerner's Granulomatosis -case report. Petkovic RT, Radovic M,Pejcic T, Rancic M, Borovac ND et al. Acta Medica Medianae. 2008; 47(3):78-81.

6. Diffuse liver uptake of technetium-99m-MDP bone scan due to hepatotoxicity secondary to methotrexate therapy. Lin K-H, Shih B-F, Tsao C-H,Wu M-C.Ann Nucl Med Sci 2005;18:111115.

7. Technetium-99m methylene diphosphonate uptake in hepatic necrosis secondary to respiratory failure. Mahajan SM, Digamber NS, Sharma R. World J Nucl.Med. 2013;12(3):116119.

8. Gentili A, Miron SD, Bellon E. Nonosseous accumulation of bone seeking radiopharmaceuticals.Radiographics.1990;10:8 71-881. 